\title{
Oxidative stress markers and lipid profiles of patients with polycystic ovary syndrome in a Nigerian tertiary hospital
}

Chukwunonso Isaiah Enechukwu ${ }^{1,2}$, Anaelechi Jude Onuegbu², Madu Japheth Olisekodiaka², George Uchenna Eleje', Joseph Ifeanyichukwu Ikechebelu', Joseph Odirichukwu Ugboaja', Ubuo Kalu Amah², John Ekenedirichukwu Okwara², Anthony Osita Igwegbe ${ }^{1}$

\footnotetext{
${ }^{1}$ Department of Obstetrics \& Gynaecology, Nnamdi Azikiwe University Teaching Hospital Nnewi, Nigeria; ${ }^{2}$ Department of Chemical Pathology, Faculty of Medicine, Nnamdi Azikiwe University, Awka, Nigeria
}

\section{Objective}

To determine the activities of oxidative stress markers and lipid profiles of patients with polycystic ovary syndrome (PCOS) in Nnamdi Azikiwe University Teaching Hospital Nnewi, Nigeria.

\section{Methods}

This was a nested case-control study consisting of 50 PCOS patients and 50 healthy women of the same age range without any evidence of PCOS. The study measured the levels of malondialdehyde (MDA), activity of superoxide dismutase (SOD), glutathione peroxidase (GSH-Px), total antioxidant capacity (TAC); concentrations of total cholesterol (TC), triglycerides (TG), and low-density lipoprotein cholesterol (LDL-C), as well as high-density lipoprotein cholesterol (HDL-C) using standard spectrophotometric methods. Anthropometric indices were also assessed. $P$-values of $<0.05$ were taken to be statistically significant.

Results

There were significantly higher levels of MDA $(P=0.002)$, lower activity of SOD $(P<0.001)$, and lower TAC $(P=0.001)$ in PCOS patients when compared with the controls. There were higher concentrations of TC $(P=0.017)$ and LDL-C $P=0.012)$ in PCOS patients than in controls. Significant differences were not observed between the 2 groups in terms of the activity of GSH-Px, as well as the concentrations of HDL-C and TG. The body mass index, waist circumference, and waist-hip ratio were all significantly higher in PCOS patients.

\section{Conclusion}

This study revealed higher levels of MDA, as well as lower activity of SOD and TAC, among PCOS patients. Furthermore, there were higher levels of TC and LDL-C among the PCOS patients. Hence, monitoring these parameters may improve the clinical management of PCOS.

Keywords: Polycystic ovary syndrome; Oxidative stress; Dyslipidaemia; Obesity; Cardiometabolic risks

\section{Introduction}

Polycystic ovary syndrome (PCOS) is an endocrine disorder affecting women of reproductive age and is a frequent cause of anovulation as well as infertility $[1,2]$. It is a complex disease entity with a wide range of symptoms and manifestations, ranging from menstrual cycle disorders (oligomenorrhea or amenorrhea); clinical and/or biochemical evidence of hyperandrogenism, to transvaginal ultrasound scan morphol-
Received: 2018.12.05. Revised: 2019.02.26. Accepted: 2019.03.18. Corresponding author: Chukwunonso Isaiah Enechukwu Department of Obstetrics \& Gynaecology, Nnamdi Azikiwe University Teaching Hospital Nnewi, Old Nnewi-Oba Road, Nnewi 435101, Nigeria

E-mail: nkystarme@gmail.com

https://orcid.org/0000-0003-4639-5127

Articles published in Obstet Gynecol Sci are open-access, distributed under the terms of the Creative Commons Attribution Non-Commercial License (http://creativecommons. org/licenses/by-nc/3.0/) which permits unrestricted non-commercial use, distribution, and reproduction in any medium, provided the original work is properly cited.

Copyright $\odot 2019$ Korean Society of Obstetrics and Gynecology 


\section{Obstetrics \& Gynecology Science}

Vol. 62, No. 5, 2019

ogy of polycystic ovaries (PCO); with the exclusion of other etiologies [3]. Over the years, diagnosis has been controversial; however the Rotterdam criteria admit at least 2 out of the 3 aforementioned features for universally accepted diagnosis [4]. PCOS is also associated with some metabolic abnormalities such as dyslipidemia, obesity, insulin resistance, type II diabetes mellitus, and hypertension [5].

It is unclear what causes PCOS; however, it has been widely linked to insulin resistance which gives rise to hyperandrogenism, dyslipidemia and other metabolic disturbances $[1,2]$. Hyperandrogenism disrupts ovarian steroidogenesis as well as folliculogenesis, leading to anovulation [1]. Other possible etiological factors for PCOS include disorders of the hypothalamic-pituitary-ovarian axis, and the patient's genetic predisposition, which varies geographically [6-10]. Presently, there appears to be a link between PCOS and oxidative stress, and whether oxidative stress is an etiology or a consequence is yet to be unravelled $[1,2,5]$.

Oxidative stress ensues when there is an imbalance between the generation of free radicals and antioxidants in biological systems, with a resultant negative shift in the equilibrium $[11,12]$. Excess free radicals, with unpaired electrons in their outermost shells react with both macro- and micromolecules of the body, oxidizing them and consequently bringing about deleterious alterations within cells, on cell membranes and receptors, proteins, lipids, lipoproteins, carbohydrates, and DNA strands [13-15].

At physiological levels; free radicals play some beneficial roles such as detoxification of pathogens, regulation of signal transduction and transcription, cell differentiation, and serving as secondary messengers for the various tissues, organs and systems of the body $[13,14]$. Reactive oxygen species, balanced with their respective antioxidant enzymes, vitamins and minerals, modulate ovulatory function leading to cyclical release of mature follicles (ova) in readiness for conception $[16,17]$. Antioxidants protect the developing follicles and ensure a homeostatic redox environment for the eggs $[16,17]$.

Superoxide dismutase (SOD) and glutathione peroxidase (GSH-Px) are among the major antioxidant enzymes which neutralize oxygen free radicals and spare the body of their damaging effects $[17,18]$. Total antioxidant capacity (TAC) is a measure of the ability of biological materials to neutralize free radicals, hence preventing the evolution or propagation of a particular disease process $[1,19]$.

Lipid peroxidation products include malondialdehyde (MDA) and hydroxyl radicals, which accumulate as a result of intracellular and cell wall damage involving polyunsaturated fatty acids, with increased levels of reactive oxygen species $[2,20]$. Hence, serum MDA levels could reflect the extent of lipid peroxidation as well as cell membrane and DNA damage [21].

There is a paucity of studies on PCOS in developing country settings. To the best of our knowledge, relationships between PCOS and oxidative stress markers, as well as lipid profiles, have never been investigated in our environment; meanwhile, the disease entity (PCOS) displays significant racial, familial, and genetic variation $[1,8,10]$.

Moreover, previous studies in developed settings documented inconsistent findings among PCOS patients in terms of activities of antioxidant enzymes and TAC. These inconsistent findings ranged from higher to lower activities, and then to no significant differences. Hence, more studies are necessary to further investigate such relationships involving antioxidants and PCOS.

Furthermore, PCOS is a chronic and multisystemic disorder; hence, wholistic management should go beyond evaluation of the reproductive problems of patients such as anovulatory infertility and irregularity of menses. The various possible metabolic risks of this syndrome should also be investigated and monitored. The outcome of this study therefore, may inform the routine assessment of lipid profiles and possibly oxidative stress markers among PCOS patients in our gynecological practice. This may guarantee improved monitoring and management of the long-term metabolic complications of this syndrome.

The aim of this study therefore, was to determine the activities of oxidative stress markers and lipid profiles of patients with PCOS in Nnamdi Azikiwe University Teaching Hospital (NAUTH) Nnewi, Nigeria.

\section{Materials and methods}

This study was conducted in NAUTH, which is a tertiary health institution in Nnewi, Anambra state, south-east of Nigeria. The subjects were selected, interviewed, and examined at the gynecology clinic. Blood samples were collected, separated, stored and analyzed at the chemical pathology laboratory of NAUTH.

The study was a nested case-control research project conducted among 50 PCOS patients and 50 healthy controls 


\section{Obstetrics \& Gynecology Science}

Chukwunonso Isaiah Enechukwu, et al. Oxidative stress markers in PCOS

of the same age range without any evidence of PCOS. The diagnosis of PCOS was made according to the Rotterdam criteria, as defined by the European Society for Human Reproduction and Embryology and the American Society for Reproductive Medicine consensus declaration of 2003 [4]. The diagnostic criteria employed at least 2 out of the following 3 features: 1) Oligo- and/or anovulation; 2) Clinical and/ or biochemical evidence of hyperandrogenism; and 3) Transvaginal ultrasound scan morphology of PCO with 12 or more follicles in each ovary measuring $2-9 \mathrm{~mm}$ in diameter and/or increased ovarian volume $>10 \mathrm{~mL}[3,4]$.

The subjects were duly informed about the study protocol using an information sheet. Only subjects who met the inclusion criteria and gave written consent were recruited. They were interviewed to obtain information on socio-demographic characteristics and relevant gynecological history, using a structured questionnaire. The eligible subjects were recruited over a period of 5 months, from 17th November 2016 to 20th April 2017. Women within reproductive age and diagnosed with PCOS according to the Rotterdam criteria were included, and these constituted the test group.

The control group were healthy women without any evidence of PCOS, recruited from members of the hospital staff and within the same age range as the test group. The study purpose and protocol were explained in detail to them with an information sheet before recruitment. Only subjects who freely gave consent were recruited for this study. The control and test subjects were reassured that participation was entirely voluntary. The ethical principles of confidentiality, subject autonomy, and non-maleficence, amongst others were strictly followed to protect both the control and test subjects. They were assured of anonymity. Their personal details were coded, no names were documented; they were assigned numbers, such that no data could be traceable to them.

The minimum sample size for each group was calculated using the formula;

$\mathrm{N}=\frac{Z^{2} P Q}{d^{2}}$ [22]; Where $\mathrm{N}=$ sample size, $\mathrm{Z}=$ standard normal deviation at $95 \%$ confidence interval, which is 1.96 , $\mathrm{Q}=$ alternate proportion (1-P), $\mathrm{d}=$ degree of precision set at $0.05, \mathrm{P}=$ proportion of the target population. In this case, prevalence rate or proportion of PCOS patients in Nnewi=2.2\% [7]. Therefore, $\mathrm{N}=33$ subjects for each group. To account for attrition; a new sample size $\mathrm{N}^{1}$ was obtained as follows: $N^{1}=$ calculated samplex $\frac{100}{100-x}$; Where $x=10 \%$ attrition. $N^{1}=36.3 \sim 37$ subjects for each group. Hence, a total of
74 subjects were needed as a minimum sample size for the study, i.e., 37 for the PCOS patients (test group) and another 37 for apparently healthy women (control group) without any evidence of PCOS and who were within the same age range. However, a total of 100 subjects were eventually recruited i.e. 50 for test group and another 50 for the control group, in order to increase the statistical power of the study.

The exclusion criteria were: PCOS patients diagnosed with diabetes mellitus, chronic hypertension, cardiovascular diseases, thyroid dysfunction, and endometriosis. Women diagnosed with PCOS who had been on hormonal therapy, lipid-lowering or insulin-sensitizing drugs over the previous 3 months were also excluded.

The body weight of each subject in kilograms $(\mathrm{kg})$ was determined using a clinical weighing scale, while the height in meters $(\mathrm{m})$ was measured using a stadiometer. Body mass index (BMI) was calculated by dividing the weight by the square of the height $\left(\mathrm{kg} / \mathrm{m}^{2}\right)$. The World Health Organization (WHO) recognizes a $\mathrm{BMI}$ of 30 and above as obesity [23]. The waist circumference (WC) in centimeters $(\mathrm{cm})$ was obtained with a non-stretchable measuring tape placed at the approximate midpoint between the lower border of the last palpable rib and the top of the iliac crest [23]. The hip circumference $(\mathrm{HC})$ in $\mathrm{cm}$ was also obtained with the nonstretchable measuring tape placed around the widest portion of the buttocks, using the greater trochanter of the femur as a landmark. The waist-hip ratio (WC:HC) was then obtained by dividing the WC by the $\mathrm{HC}$. The WHO recognizes a WC:HC ratio of greater than 0.85 as abdominal obesity in women. ${ }^{23}$ It also recognizes a cut-off point of $80 \mathrm{~cm}$ for WC of females who are at increased risk of metabolic complications, and $88 \mathrm{~cm}$ for those at substantially increased risk [23]. The blood pressure of each subject was measured using a mercury sphygmomanometer and stethoscope.

\section{Blood collection and analysis}

Five $\mathrm{mL}$ of venous blood was collected aseptically from each subject into a plain tube using a sterile syringe and needle, from the ante-cubital vein of one of the arms, after an overnight fast of approximately 12-14 hours. The blood sample obtained was centrifuged at 3,000 revolutions per minute for 15 minutes. The serum derived from the blood was stored at $-86^{\circ} \mathrm{C}$ and subsequently used to estimate the levels of total cholesterol (TC), triglycerides (TG), low-density lipoprotein cholesterol (LDL-C), high-density lipoprotein cholesterol 


\title{
Obstetrics \& Gynecology Science
}

\author{
Vol. 62, No. 5, 2019
}

(HDL-C) and MDA, as well as the activities of SOD, GSH-Px, and TAC. An enzymatic colorimetric method was used to assay the levels of $\mathrm{TC}, \mathrm{TG}$, and $\mathrm{HDL}-\mathrm{C}$, with their respective absorbances read by a spectrophotometer. LDL-C was determined using Friedewald's formula. The level of MDA was determined as a measure of thiobarbituric acid reacting substances. The activity of GSH-Px was determined using dithiodinitrobenzoic acid; while SOD activity was estimated using the principle of inhibition of auto-oxidation of adrenaline. TAC was estimated via the ferric reducing ability of plasma method.

\section{Statistical analysis}

All data obtained from the study were analyzed using the Statistical Package for Social Sciences (SPSS) Windows version 21.0 software (IBM Corp., Armonk, NY, USA, 2012). Values are presented as means \pm standard deviation. Student's t-test was used to test for statistical significance between the test group and the control group. Relationships between variables were assessed using Pearson's correlation coefficient. The results were regarded as significant at $P<0.05$.

\section{Results}

Fifty-five PCOS patients and 59 healthy controls were assessed for eligibility. Of these, 5 PCOS patients and 9 controls were excluded, hence 50 test subjects and 50 controls were enrolled in the study (Fig. 1).

Results from this study revealed significantly higher values for weight, BMI, WC, WC:HC ratio and diastolic blood pressure in the test group than the control group (Table 1). There were no significant differences in age, height, $\mathrm{HC}$ and systolic blood pressure between the test group and the control group (Table 1).

The mean serum level of MDA was significantly higher in women with PCOS than in the control group, while the mean activity of SOD and TAC were significantly lower in the test group than the control group (Table 1). No significant difference was observed in the GSH-Px activity between the test group and the control group (Table 1).

The mean serum levels of TC and LDL-C were significantly higher in the test group than the control group (Table 1). Significant differences were not observed in the mean TG and $\mathrm{HDL}-\mathrm{C}$ between the test group and the control group (Table 1).

Oxidative stress markers according to central obesity in control and test groups showed no significant difference (Table 2).

The oxidative stress markers and lipid profile according to the menstrual cycle showed no significant difference among the control subjects (Table 3).

Among the 50 PCOS patients, 32 (64\%) had frank PCOS; $8(16 \%)$ had ovulatory PCOS; 7 (14\%) were normoandrogenic, while $3(6 \%)$ were non-PCO PCOS patients.

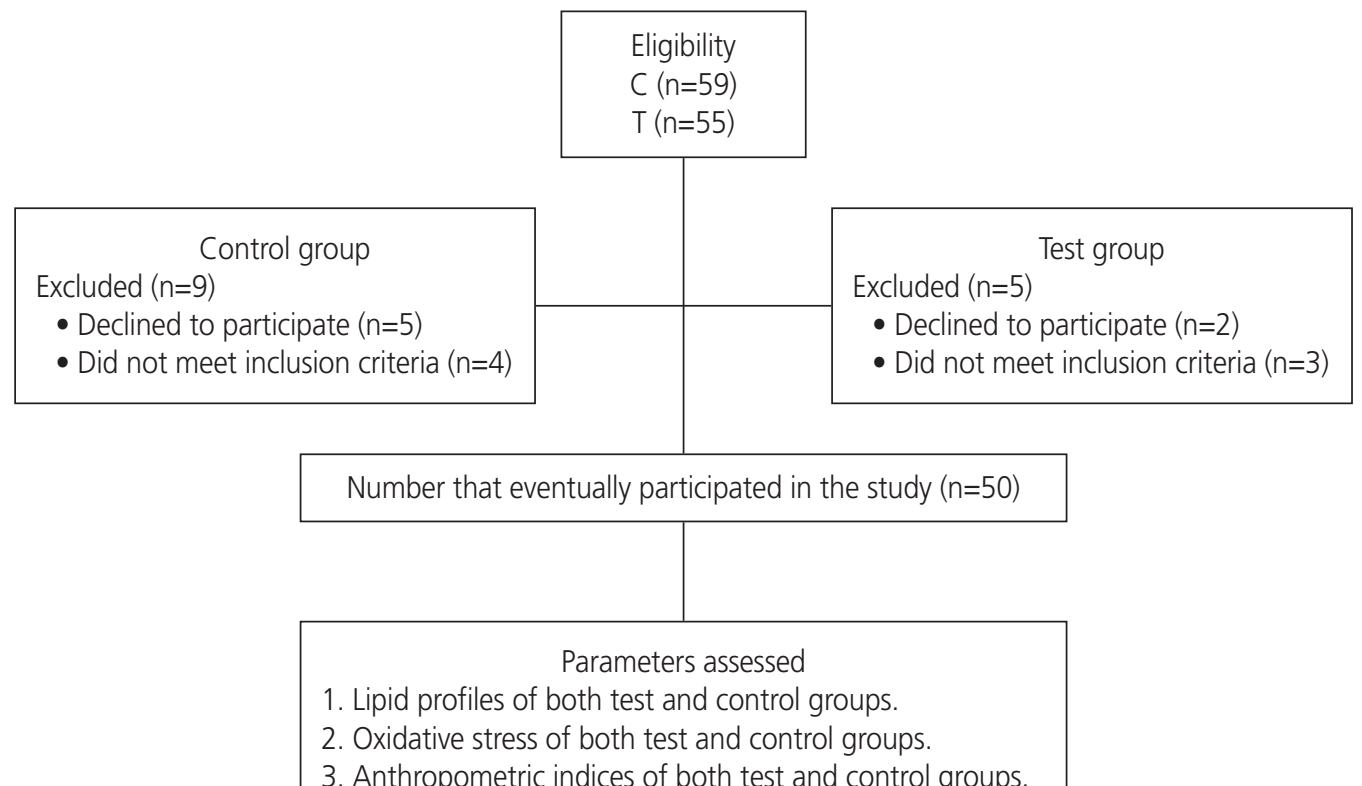

Fig. 1. Flow pattern of the study. 


\section{Obstetrics \& Gynecology Science}

Chukwunonso Isaiah Enechukwu, et al. Oxidative stress markers in PCOS

Table 1. Mean \pm standard deviation (SD) of age, blood pressure, anthropometrics; serum levels of fasting lipid profile; malondialdehyde (MDA), superoxide dismutase (SOD), glutathione peroxidase (GSH-Px), and total antioxidant capacity (TAC) in polycystic ovary syndrome women and control group

\begin{tabular}{|c|c|c|c|c|}
\hline Parameters & Control group $(n=50)$ & Test group $(n=50)$ & $t$-test & $P$-value \\
\hline Age (yr) & $27.80 \pm 5.11$ & $28.18 \pm 5.06$ & -0.374 & 0.710 \\
\hline $\mathrm{SBP}(\mathrm{mmHg})$ & $111.72 \pm 9.36$ & $113.60 \pm 8.98$ & -1.025 & 0.308 \\
\hline $\mathrm{DBP}(\mathrm{mmHg})$ & $68.80 \pm 11.72$ & $73.00 \pm 6.78$ & -2.194 & $0.031^{a)}$ \\
\hline Weight (kg) & $65.61 \pm 10.13$ & $72.41 \pm 15.44$ & -2.604 & $0.011^{a)}$ \\
\hline Height (kg) & $1.64 \pm 0.08$ & $1.65 \pm 0.08$ & -0.763 & 0.447 \\
\hline $\mathrm{BMI}\left(\mathrm{kg} / \mathrm{m}^{2}\right)$ & $24.40 \pm 2.86$ & $26.58 \pm 5.16$ & -2.616 & $0.010^{a)}$ \\
\hline$W C(\mathrm{~cm})$ & $81.86 \pm 8.20$ & $88.73 \pm 12.19$ & -3.306 & $0.001^{a)}$ \\
\hline $\mathrm{HC}(\mathrm{cm})$ & $99.04 \pm 8.34$ & $100.58 \pm 10.41$ & -0.816 & 0.416 \\
\hline $\mathrm{TC}(\mathrm{mmol} / \mathrm{L})$ & $4.44 \pm 0.63$ & $4.93 \pm 1.27$ & -2.418 & $0.017^{\mathrm{a})}$ \\
\hline $\mathrm{TG}(\mathrm{mmol} / \mathrm{L})$ & $0.90 \pm 0.32$ & $1.08 \pm 0.58$ & -1.960 & 0.053 \\
\hline $\mathrm{HDL}-\mathrm{C}$ (mmol/L) & $0.92 \pm 0.20$ & $0.85 \pm 0.28$ & 1.537 & 0.128 \\
\hline LDL-C (mmol/L) & $3.10 \pm 0.63$ & $3.57 \pm 1.13$ & -2.556 & $0.012^{\mathrm{a})}$ \\
\hline $\mathrm{SOD}(\mathrm{U} / \mathrm{L})$ & $12.27 \pm 1.75$ & $8.63 \pm 4.17$ & 5.680 & $<0.001^{a)}$ \\
\hline $\mathrm{MDA}(\mathrm{nmol} / \mathrm{mL})$ & $2.34 \pm 0.47$ & $2.77 \pm 0.81$ & -3.209 & $0.002^{a)}$ \\
\hline GSH-Px (U/mL) & $0.74 \pm 0.13$ & $0.71 \pm 0.12$ & 0.987 & 0.326 \\
\hline TAC ( $\mu \mathrm{mol} / \mathrm{L})$ & $1001.73 \pm 171.23$ & $881.20 \pm 174.06$ & 3.491 & $0.001^{a)}$ \\
\hline
\end{tabular}

Data are shown as mean \pm SD.

SBP, systolic blood pressure; DBP, diastolic blood pressure; BMI, body mass index; WC, waist circumference; HC, hip circumference; TC, total cholesterol; TG, triglyceride; HDL-C, high-density lipoprotein cholesterol; LDL-C, low-density lipoprotein cholesterol.

${ }^{\text {a) }} P<0.05$.

Table 2. Oxidative stress markers according to central obesity in control and test groups

\begin{tabular}{lcccccc}
\hline \multirow{2}{*}{ Parameters } & \multicolumn{2}{c}{$\begin{array}{c}\text { Control group } \\
(\mathbf{n}=\mathbf{5 0})\end{array}$} & & \multicolumn{2}{c}{ Test group $(\mathbf{n = 5 0 )}$} \\
\cline { 2 - 3 } \cline { 6 - 7 } & r value & $\boldsymbol{P}$-value & & r value & $\boldsymbol{P}$-value \\
\hline WC:HC vs. SOD & 0.109 & 0.449 & & -0.036 & 0.803 \\
WC:HC vs. MDA & 0.118 & 0.414 & & -0.101 & 0.485 \\
WC:HC vs. GSH-Px & -0.143 & 0.323 & & 0.130 & 0.367 \\
WC:HC vs. TAC & 0.011 & 0.940 & & -0.109 & 0.450 \\
\hline
\end{tabular}

WC, waist circumference; $H C$, hip circumference; SOD, superoxide dismutase; MDA, malondialdehyde; GSH-Px, glutathione peroxidase; TAC, total antioxidant capacity.

\section{Discussion}

In this study, mean serum MDA levels were significantly higher in PCOS patients than in the corresponding control group. The activity of SOD and TAC were significantly lower in PCOS patients when compared with the controls. There was no significant difference seen in the activity of GSH-Px in the test and control groups. Significantly higher levels of TC and LDL-C were obtained in the PCOS patients when compared with the controls. No significant difference was seen in the mean HDL-C and TG concentrations in the test and control groups. The study also revealed significantly higher values for body weight, BMI, WC, and WC: HC ratio in the test group compared to the control group. There was no significant difference in age, height, and HC between the test group and the control group.

This study revealed that mean serum MDA levels were significantly higher in PCOS patients than the controls. This finding corroborates other works $[2,12,20,24]$. MDA is an indicator of chronic oxidative states, which may be pathologically found in PCOS patients. Insulin resistance, hyperandrogenism, dyslipidemia and obesity associated with PCOS possibly increase the levels of MDA and at the same time decrease antioxidant enzyme levels $[2,12]$. The serum levels of MDA reflect the extent of lipid peroxidation and tissue damage ongoing in PCOS patients $[20,21]$. Even among nonobese PCOS patients, levels of MDA have also been found to 


\title{
Obstetrics \& Gynecology Science
}

\author{
Vol. 62 , No. 5, 2019
}

Table 3. Oxidative stress markers and lipid profile according to menstrual cycle in control group

\begin{tabular}{|c|c|c|c|c|c|}
\hline Parameters & Follicular & Ovulatory & Luteal & f-test & $P$-value \\
\hline SOD & $9.17 \pm 4.52$ & $7.50 \pm 1.18$ & $8.33 \pm 4.26$ & 0.419 & 0.660 \\
\hline MDA & $2.76 \pm 0.79$ & $2.56 \pm 0.71$ & $2.82 \pm 0.88$ & 0.206 & 0.815 \\
\hline GSH-Px & $0.71 \pm 0.08$ & $0.74 \pm 0.07$ & $0.71 \pm 0.16$ & 0.199 & 0.820 \\
\hline TAC & $862.68 \pm 201.92$ & $961.30 \pm 87.28$ & $882.20 \pm 156.92$ & 0.651 & 0.526 \\
\hline $\mathrm{TC}$ & $4.99 \pm 1.26$ & $5.78 \pm 1.14$ & $4.66 \pm 1.26$ & 1.676 & 0.198 \\
\hline TG & $1.18 \pm 0.75$ & $0.91 \pm 0.21$ & $1.02 \pm 0.40$ & 0.688 & 0.507 \\
\hline HDL-C & $0.92 \pm 0.28$ & $0.83 \pm 0.15$ & $0.78 \pm 0.29$ & 1.457 & 0.243 \\
\hline LDL-C & $3.53 \pm 1.05$ & $4.54 \pm 0.95$ & $3.39 \pm 1.17$ & 2.260 & 0.116 \\
\hline
\end{tabular}

Data are shown as mean \pm standard deviation.

SOD, superoxide dismutase; MDA, malondialdehyde; GSH-Px, glutathione peroxidase; TAC, total antioxidant capacity; HDL-C, high-density lipoprotein cholesterol; LDL-C, low-density lipoprotein cholesterol; TC, total cholesterol; TG, triglycerides.

be significantly higher than in controls $[12,25,26]$. MDA also constitutes a risk factor for cardiovascular diseases $[12,20]$.

Furthermore, in this study, the mean SOD activity for the test group was significantly lower than that of the control group. This finding is in agreement with some previous studies $[24,27]$, but not with certain other works $[20,25]$ that demonstrated significantly higher SOD activity in PCOS patients, when compared with control groups. The authors actually concluded that the elevations in SOD activity recorded in those studies might have been due to a compensatory response by the body's defense mechanisms to higher circulating levels of oxidants [25].

SOD mounts a defense against reactive oxygen species, hence catalyzing the conversion of superoxide to an intermediary product, hydrogen peroxide [21]. It is therefore expedient that hydrogen peroxide be cleared rapidly from the system using GSH-Px $[21,24]$. The current study did not observe any significant difference in mean activity of GSH-Px among PCOS patients when compared with controls. This particular finding has also been documented previously [20]. However; a number of other authors demonstrated significantly lower activity of GSH-Px among obese PCOS patients, when compared with other obese women without PCOS [27]. GSHPx eliminates peroxides in biological systems by employing a reduced form of glutathione as a hydrogen ion donor [16].

This study also revealed that TAC was significantly lower in the test group than the control group. This finding corroborates previous work [28] and demonstrates significantly lower TAC means in PCOS patients. On the other hand, other studies showed significantly higher TAC means in PCOS patients than in controls [25]. This could also be explained by compensation in response to high circulating levels of oxidants. Nevertheless, Yilmaz and colleagues did not observe any significant difference in TAC means between their test and control groups [26].

In this study, TC and LDL-C levels were significantly higher in PCOS patients than the control group. These findings are in agreement with previous published studies which showed similar patterns of dyslipidemia, with significantly higher levels of TC and LDL-C in PCOS patients than the controls [5,29-32]. However, significant differences were not observed in the current study in the mean HDL-C and TG concentrations, unlike the previously cited studies above which demonstrated significantly lower levels of HDL-C and higher levels of TG in the test groups, when compared with the control groups. Dyslipidemia could be viewed as a possible complication of PCOS, with abnormalities in lipid and lipoprotein metabolism $[5,29]$, and has been associated with insulin resistance and central obesity $[29,33,34]$, which was also significantly higher among PCOS patients in the current study. Insulin resistance limits the ability of insulin to suppress lipolysis, hence promoting an enormous release of free fatty acids from adipose tissues into the systemic circulation [31]. It also encourages a high hepatic load of TG and very LDL-C, with subsequent conversion to LDL-C [32]. In association with other variables, dyslipidemia constitutes a significant risk factor for cardiometabolic diseases [5,29-33].

The American College of Obstetricians and Gynecologists as well as the Androgen Excess and PCOS Society recommend that a complete fasting lipid and lipoprotein evaluation 


\section{Obstetrics \& Gynecology Science}

Chukwunonso Isaiah Enechukwu, et al. Oxidative stress markers in PCOS

be done for all PCOS patients as part of their cardiovascular risk assessment $[30,31,35,36]$. To this effect, the primary target remains $L D L-C$, being the most atherogenic lipoprotein biomolecule [5,30-33]. Following the assessment of PCOS patients for dyslipidemia, those with normal fasting lipid profiles will require a repeat assessment in 2 years' time, or earlier in the presence of an increase in body weight $[30,31,35]$. Dietary adjustments and physical exercise, as part of lifestyle modification, represent the first line therapy for all patients with PCOS, especially those with dyslipidemia [30,31,35,37]. The use of lipid lowering drugs such as statins may be desirable when lifestyle modification fails to correct the dyslipidemia $[5,33]$. Insulin sensitizing drugs such as metformin may also be beneficial $[35,36]$.

The strength of this study lies in the fact that confounders of oxidative stress and dyslipidemia were excluded among the PCOS patients and the controls. These confounders included endometriosis, thyroid dysfunction, cardiovascular diseases, diabetes mellitus, and chronic hypertension. Subjects diagnosed with PCOS, as well as controls who have undergone hormonal therapy, or used lipid-lowering or insulin-sensitizing drugs over the previous 3 months were also excluded. Furthermore, the controversies and lingering debates surrounding the diagnosis of PCOS were avoided by adopting the Rotterdam criteria in this study.

One of the limitations of this study was that we could not analyze oxidative stress markers and lipid profiles according to the 4 subclasses of PCOS (frank, ovulatory, non-PCO, and normoandrogenic) due to the small sample size. There was no even distribution of the test subjects among the 4 subclasses of PCOS, as majority of the test subjects had frank PCOS. This is, therefore, a subject for further study. Another limitation of this study was that only one oxidant marker was assessed, while the others were antioxidants.

In conclusion, this study showed higher levels of MDA as well as lowered activity of SOD and TAC among PCOS patients. Furthermore, there were higher levels of TC and LDL-C among the PCOS patients. Hence, monitoring these parameters may improve the management of PCOS.

Recommendations: evaluation of PCOS patients in the clinic, irrespective of their reproductive disorders; should incorporate assessment of lipid profiles and possibly oxidative stress markers. Interpretation of the results of these analytes may necessitate possible referral to cardiologists for further assessment of cardiovascular disease risks in these patients.
Invariably, this will go a long way in encouraging collaboration and multidisciplinary approaches for wholistic management of patients with this multisystemic disorder, especially for those living in this environment.

Furthermore, the assessment of obesity in PCOS patients should routinely incorporate WC and WC:HC ratio indices, in addition to BMI.

\section{Acknowledgements}

We wish to thank Dr. Christian Onah, Principal Medical Laboratory Scientist in the Department of Chemical Pathology, Nnamdi Azikiwe University Teaching Hospital Nnewi, Nigeria; for his laboratory assistance.

\section{Conflict of interest}

No potential conflict of interest relevant to this article was reported.

\section{Ethical approval}

The study was approved by the Institutional Review Board of Nnamdi Azikiwe University Teaching Hospital, Nnewi (IRB No. NAUTH/CS/66NOL.9/99; dated: 16th November 2016) and performed in accordance with the principles of the Declaration of Helsinki. Written informed consents were obtained.

\section{Patient consent}

The patients provided written informed consent for the publication and the use of their images.

\section{References}

1. Lee JY, Baw CK, Gupta S, Aziz N, Agarwal A. Role of oxidative stress in polycystic ovary syndrome. Curr Womens Health Rev 2010;6:96-107.

2. Karabulut AB, Cakmak M, Kiran RT, Sahin I. Oxidative stress status, metabolic profile and cardiovascular risk 


\section{Obstetrics \& Gynecology Science}

Vol. 62, No. 5, 2019

factors in patients with polycystic ovary syndrome. Med Sci (Turkey) 2012;1:27-34.

3. Balen AH, Laven JS, Tan SL, Dewailly D. Ultrasound assessment of the polycystic ovary: international consensus definitions. Hum Reprod Update 2003;9:505-14.

4. Rotterdam ESHRE/ASRM-sponsored PCOS consensus workshop group. Revised 2003 consensus on diagnostic criteria and long-term health risks related to polycystic ovary syndrome. Fertil Steril 2004;81:19-25.

5. Macut D, Bjekić-Macut J, Savić-Radojević A. Dyslipidemia and oxidative stress in PCOS. Front Horm Res 2013;40:51-63.

6. Chen X, Yang D, Mo Y, Li L, Chen Y, Huang Y. Prevalence of polycystic ovary syndrome in unselected women from southern China. Eur J Obstet Gynecol Reprod Biol 2008;139:59-64.

7. Igwegbe AO, Eleje GU, Enechukwu Cl. Polycystic ovary syndrome: a review of management outcomes in a low resource setting. J Womens Health Issues Care 2013;2:3.

8. Balen A, Michelmore K. What is polycystic ovary syndrome? Are national views important? Hum Reprod 2002;17:2219-27.

9. Hussein B, Alalaf S. Prevalence and characteristics of polycystic ovarian syndrome in a sample of infertile Kurdish women attending IVF infertility center in maternity teaching hospital of Erbil City. Open J Obstet Gynecol 2013;3:577-85.

10. Joe-kechebelu NN, Mbamara SU, Ikechebelu JI. Familial trend in polycystic ovarian syndrome: report of two cases. Ann Afr Med 2013;12:182-4.

11. Betteridge DJ. What is oxidative stress? Metabolism 2000;49:3-8.

12. Desai V, Prasad NR, Manohar SM, Sachan A, Narasimha $S R$, Bitla AR. Oxidative stress in non-obese women with polycystic ovarian syndrome. J Clin Diagn Res 2014;8:CC01-03.

13. Yoshikawa T, Naito Y. What is oxidative stress? Japan Med Assoc J 2002;45:271-6.

14. Valko M, Leibfritz D, Moncol J, Cronin MT, Mazur M, Telser J. Free radicals and antioxidants in normal physiological functions and human disease. Int J Biochem Cell Biol 2007;39:44-84.

15. Vincent HK, Taylor AG. Biomarkers and potential mechanisms of obesity-induced oxidant stress in humans. Int J Obes 2006;30:400-18.
16. Agarwal A, Aponte-Mellado A, Premkumar BJ, Shaman A, Gupta S. The effects of oxidative stress on female reproduction: a review. Reprod Biol Endocrinol 2012;10:49.

17. Agarwal A, Gupta S, Sharma RK. Role of oxidative stress in female reproduction. Reprod Biol Endocrinol 2005;3:28.

18. Aguilar A, Alvarez-Vijande R, Capdevila S, Alcoberro J, Alcaraz A. Antioxidant patterns (superoxide dismutase, glutathione reductase, and glutathione peroxidase) in kidneys from non-heart-beating-donors: experimental study. Transplant Proc 2007;39:249-52.

19. Rubio CP, Hernández-Ruiz J, Martinez-Subiela S, Tvarijonaviciute A, Ceron JJ. Spectrophotometric assays for total antioxidant capacity (TAC) in dog serum: an update. BMC Vet Res 2016;12:166.

20. Sabuncu T, Vural H, Harma M, Harma M. Oxidative stress in polycystic ovary syndrome and its contribution to the risk of cardiovascular disease. Clin Biochem 2001;34:407-13.

21. Wang HF, Zhong XH, Shi WY, Guo B. Study of malondialdehyde (MDA) content, superoxide dismutase (SOD) and glutathione peroxidase (GSH-Px) activities in chickens infected with avian infectious bronchitis virus. Afr J Biotechnol 2011;10:9213-7.

22. Fisher RA. The logic of inductive inference. J R Stat Soc 1935;98:39-82.

23. World Health Organization. Waist circumference and waist-hip ratio: report of a WHO expert consultation, Geneva, 8-11 December 2008 [Internet]. Geneva: World Health Organization; c2011 [cited 2017 May 18]. Available from: http://www.who.int/iris/handle/10665/44583.

24. Zhang $D$, Luo WY, Liao $H$, Wang $C F$, Sun $Y$. The effects of oxidative stress to PCOS. Sichuan Da Xue Xue Bao Yi Xue Ban 2008;39:421-3.

25. Kuşçu NK, Var A. Oxidative stress but not endothelial dysfunction exists in non-obese, young group of patients with polycystic ovary syndrome. Acta Obstet Gynecol Scand 2009;88:612-7.

26. Yilmaz N, Inal HA, Gorkem U, Sargin Oruc A, Yilmaz S, Turkkani A. Follicular fluid total antioxidant capacity levels in PCOS. J Obstet Gynaecol 2016;36:654-7.

27. Dos Santos ACS, Azevedo GD, Lemos TMAM. The influence of oxidative stress in inflammatory process and insulin resistance in obese women with polycystic ovary 


\section{Obstetrics \& Gynecology Science}

Chukwunonso Isaiah Enechukwu, et al. Oxidative stress markers in PCOS

syndrome. Transl Biomed 2016;7:4.

28. Fadhil JA, Nada NA, Wasan GA. Total antioxidant capacity and homocysteine levels in obese women with polycystic ovary syndrome. Int J Pharm Pharm Res 2017;8:78-86.

29. Manikkumar R, Dinesh Roy D, Krishnan V, Vijayakumar T. Association of DNA damage and dyslipidemia with polycystic ovarian syndrome. J Med Allied Sci 2013;3:15-21.

30. Wild RA, Rizzo M, Clifton S, Carmina E. Lipid levels in polycystic ovary syndrome: systematic review and metaanalysis. Fertil Steril 2011;95:1073-1079.e1-11.

31. Wild RA, Carmina E, Diamanti-Kandarakis E, Dokras A, Escobar-Morreale HF, Futterweit W, et al. Assessment of cardiovascular risk and prevention of cardiovascular disease in women with the polycystic ovary syndrome: a consensus statement by the Androgen Excess and Polycystic Ovary Syndrome (AE-PCOS) Society. J Clin Endocrinol Metab 2010;95:2038-49.

32. Ahmed Alobaidi AH, Abrahem MK, Ahmad SS, Alsama- rai AM. Dyslipidemia and oxidative stress in Iraqi women with poly cystic ovary syndroms. World J Pharm Pharm Sci 2015;4:39-48.

33. Kim JJ, Choi YM. Dyslipidemia in women with polycystic ovary syndrome. Obstet Gynecol Sci 2013;56:137-42.

34. Balen A. The pathophysiology of polycystic ovary syndrome: trying to understand PCOS and its endocrinology. Best Pract Res Clin Obstet Gynaecol 2004;18:685706.

35. Mosca L. Guidelines for prevention of cardiovascular disease in women: a summary of recommendations. Prev Cardiol 2007;10 Suppl 4:19-25.

36. ACOG Committee on Practice Bulletins--Gynecology. ACOG practice bulletin No. 108: polycystic ovary syndrome. Obstet Gynecol 2009;114:936-49.

37. Domecq JP, Prutsky G, Mullan RJ, Hazem A, Sundaresh $V$, Elamin $M B$, et al. Lifestyle modification programs in polycystic ovary syndrome: systematic review and metaanalysis. J Clin Endocrinol Metab 2013;98:4655-63. 